BNL-108290-2015-JA

\title{
Spontaneous emergence of autocatalytic information-coding polymers
}

\author{
Alexei V. Tkachenko1, a) and Sergei Maslov²,3, b) \\ 1) Center for Functional Nanomaterials, Brookhaven National Laboratory, \\ Upton NY 11973 \\ ${ }^{2)}$ Biological, Environmental and Climate Sciences Department, \\ Brookhaven National Laboratory, Upton NY 11973 \\ 3) Department of Bioengineering, University of Illinois at Urbana-Champaign, \\ 1270 Digital Computer Laboratory, MC-278 Urbana, IL 61801
}

Self-replicating systems based on information-coding polymers are of crucial importance in biology. They also recently emerged as a paradigm in material design on nano- and micro-scales. We present a general theoretical and numerical analysis of the problem of spontaneous emergence of autocatalysis for heteropolymers capable of template-assisted ligation driven by cyclic changes in the environment. Our central result is the existence of the first order transition between the regime dominated by free monomers and that with a self-sustaining population of sufficiently long chains. We provide a simple, mathematically tractable model supported by numerical simulations, which predicts the distribution of chain lengths and the onset of autocatalysis in terms of the overall monomer concentration and two fundamental rate constants. Another key result of our study is the emergence of the kineticallylimited optimal overlap length between a template and each of its two substrates. The template-assisted ligation allows for heritable transmission of the information encoded in chain sequences thus opening up the possibility of long-term memory and evolvability in such systems.

\footnotetext{
a)Electronic mail: oleksiyt@bnl.gov

b) Electronic mail: ssmaslov@gmail.com
} 


\section{INTRODUCTION}

Life as we know it today depends on self-replication of information-coding polymers. Their emergence from non-living matter is one of the greatest mysteries of fundamental science. In addition, the design of artificial self-replicating nano- and micro-scale systems is an exciting field with potential engineering applications ${ }^{1,2}$. The central challenge in both of these fields is to come up with a simple physically-realizable system obeying laws of thermodynamics, yet ultimately capable of Darwinian evolution when exposed to non-equilibrium driving forces. Chemical networks of molecules engaged in mutual catalysis is a popular candidate for such a system $^{3-6}$. Information-coding heteropolymers In fact, the most successful experimental realization of an autonomous self-replicating system involves a set of mutually catalyzing RNA-based enzymes (ribozymes) ${ }^{7}$ that show evolution-like behavior ${ }^{8}$. This is viewed as a major evidence for RNA-world hypothesis (see e.g. Refs. 9-11. )

The ribozyme activity requires relatively long polymers made of hundreds of nucleotides with carefully designed sequences. Polymers of sufficient length can be generated e.g. by traditional reversible step-growth polymerization that combines random concatenation and fragmentation of polymer chains. Furthermore, the polymer length in this type of process can be drastically increased in non-equilibrium settings such as temperature gradients ${ }^{12}$. However, even when long chains are formed, the probability of the spontaneous emergence of a sequence with an enzymatic activity remains vanishingly small, due to the exponentially large number of possible sequences.

Thus there is a strong need for a mechanism that combines the emergence of long chains with dramatic reduction of informational entropy of the sequence population. A promising candidate for such mechanism is provided by template-assisted ligation. In this process pairs of polymers are brought together by hybridization with a complementary polymer chain serving as the template and eventually ligated to form a longer chain. Unlike the nontemplated reversible step-growth polymerization used in Ref. 12, this mechanism naturally involves the information transmission from a template to the newly-ligated chain, thus opening an exciting possibility of long-term memory and evolvability. An early model involving template-assisted polymerization was proposed by P. W. Anderson and colleagues ${ }^{13,14}$. It also has been subject of more recent experimental and theoretical studies ${ }^{15,17}$. In particular, in Ref. 15 it has been demonstrated that, for a specific choice of parameters, a combination 
of non-template and template-assisted ligation can lead to the emergence of long (around 100 monomers) oligonucleotides.

In this work we carried out the theoretical and numerical analysis of a generic system in which the polymerization is driven solely by template-assisted ligation. Unlike in the models with significant contribution of non-templated concatenation, the emergence of long chains in our system represents a non-trivial chicken-or-egg problem. Indeed, the formation of long chains depends on the presence of other chains serving as templates.

In our model the "primordial soup" of monomers is driven out of equilibrium by cyclic changes in physical conditions such as temperature, salt concentration, pH, etc. (see Fig. 1ab). Polymerization occurs during the "night" phase of each cycle when the existing heteropolymers serve as templates for formation of progressively longer chains. During the "day" phase of each cycle all multi-chain structures separate and the system returns to the state of dispersed individual polymers.

We consider a general case of information-coding heteropolymers composed of $z$ types of monomers capable of making $z / 2$ mutually complementary pairs. For example, RNA is made of $z=4$ monomers forming 2 complementary pairs $A-U$ and $C-G$ responsible for doublestranded RNA structure. Similarly, we assume that hybridization between complementary segments of our generalized polymers results in formation of a double-stranded structure. During the night phase of each cycle chains form a variety of hybridized complexes. The ligation takes place in a special type of such complexes shown in Fig. 1b. The end groups of two "substrate" chains $S_{1}$ and $S_{2}$ are positioned next to each other by the virtue of hybridization with the third, "template" polymer $T$. Once the substrates are properly positioned, the new covalent bond joining them together is formed at a constant rate. We further assume that each of the intra-polymer bonds can spontaneously break at a constant rate making the overall fragmentation rate of a chain proportional to its length. If one was to leave a mixture of polymers in the night phase long enough, hybridization of multiple chains would result in the formation of a gel-like aggregate shown in Fig. 1c, effectively stopping ligation. During the day phase of the cycle (Fig. 1a) all structures of hybridized polymers dissociate while keeping their stronger internal bonds intact. Thus the day phase plays the role of the "reset" returning the system to a mixture of free polymers ready for the next night phase.

One of the major assumptions used in our study is the Random Sequence Approximation 


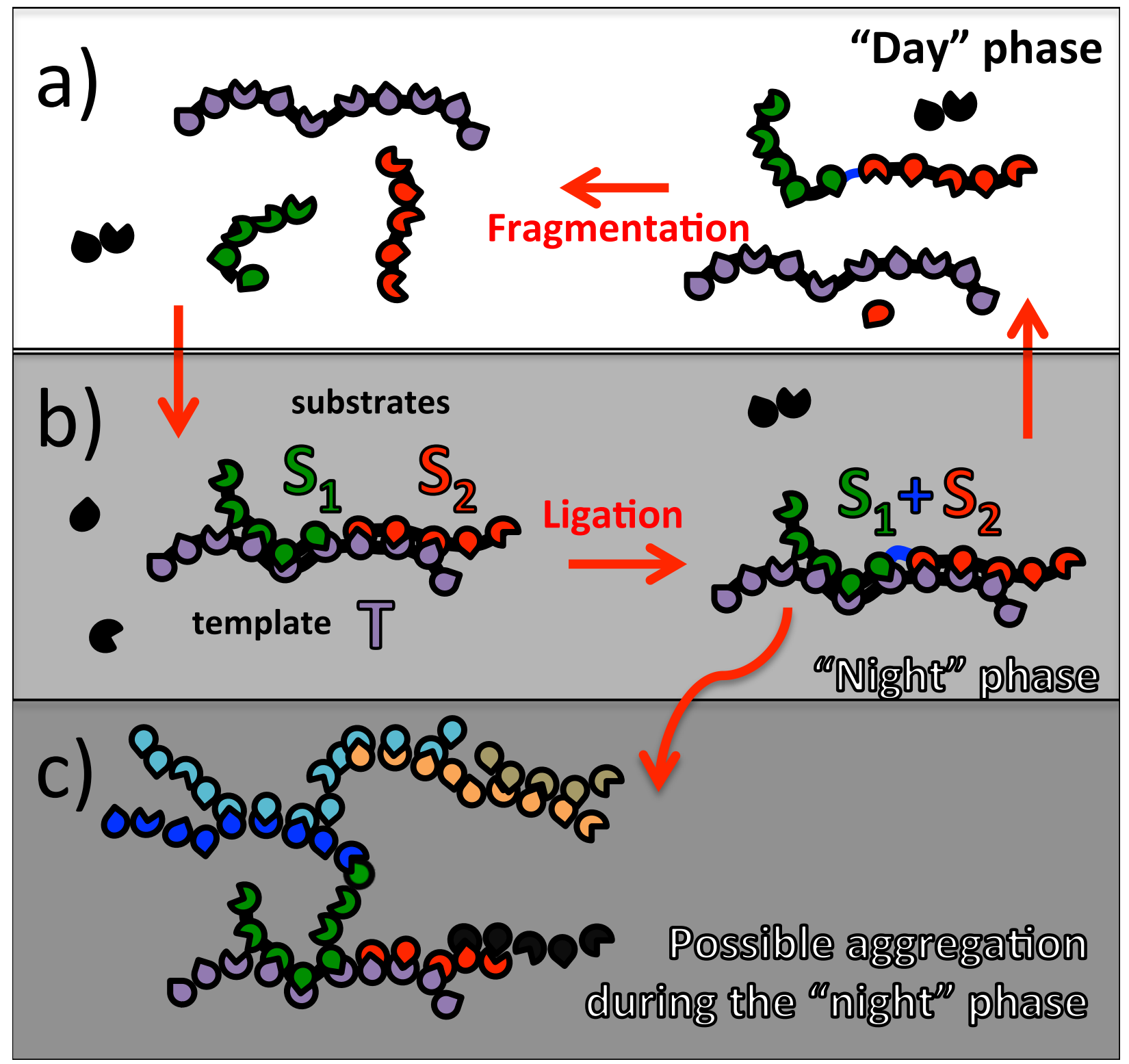

FIG. 1. The schematic representation of fundamental processes in our system. a) The "day" phase during which all hybridized complexes between heteropolymers dissociate and ligation completely stops, while fragmentation continues in all phases of the cycle. b) The "night" phase during which some polymer chains hybridize and then undergo template-assisted ligation. The ends of substrates $S_{1}$ (green) and $S_{2}$ (red) hybridized with a template $T$ (purple) are ligated at a constant rate with the newly formed bond shown in blue. c) If the "night" phase is sufficiently long heteropolymers enter the aggregation regime in which ligation effectively stops. 
(RSA) according to which each monomer in every chain can be of any type with equal probability $1 / z$. On the one hand, the RSA greatly simplifies the problem and allows us to get a concise analytical solution. On the other hand, in order to understand the transmission of sequence-encoded information and the long-term memory in our system this approximation need to be relaxed in future studies.

\section{RESULTS}

\section{A. Optimal overlap length $k_{0}$}

In general the interaction strength between any two chain segments increases with the overlap length $k$ of the region over which they are complementary to each other. Here we assume a simple linear relationship in which the binding free energy is given by $\Delta G_{0}+k \cdot \Delta G$, where $\Delta G$ is the (negative) binding free energy between two complementary monomers, while $\Delta G_{0}$ is the initiation free energy.

The equilibrium hybridization probability emerges out of the competition between two opposing kinetic processes of association and dissociation. On the one hand, the association rate exponentially decreases with the overlap length $k$ since the probability of finding a pair of polymers with complementary sequences of length $k$, is proportional to $1 / z^{k}$. On the other hand, the dissociation rate between a substrate and its template also exponentially decreases with $k$ as $\exp \left(-k \cdot \Delta G / k_{B} T\right)$ due to greater thermodynamic stability of longer complementary duplexes. The net result is that the hybridization probability is proportional to $\exp (k \cdot \epsilon)$, where

$$
\epsilon=-\Delta G / k_{B} T-\log (z)
$$

is the effective parameter combining thermodynamic and combinatorial factors. Templateassisted ligation happens at appreciable rates only for $\epsilon>0$, i.e. when $\Delta G<-k_{B} T \log (z)$. For a finite time window $t$ only the duplexes with short overlaps will reach this equilibrium.

Duplexes with longer overlaps have lifetimes much longer than $t$. Thus for them the hybridization probability is limited by the association rate alone $\sim 1 / z^{k}$. Therefore, the overall hybridization probability as a function of $k$ is strongly peaked (see Fig. 2 and Appendix A for details). As time $t$ increases, this peak slowly (logarithmically in $t$ ) shifts towards larger values of $k$ with its final value $k_{0}$ set by either the end of the night phase or (in case of long 


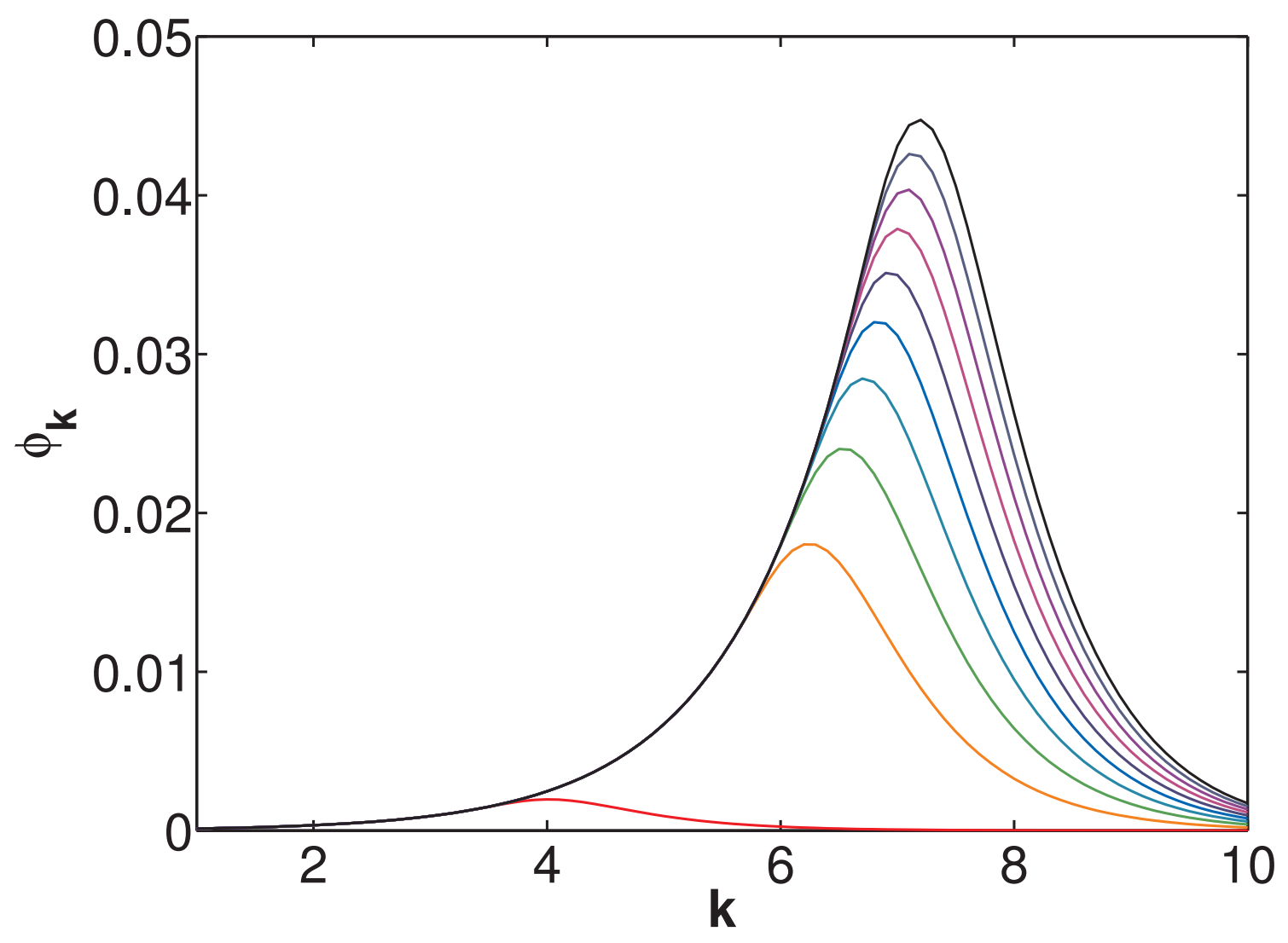

FIG. 2. Time evolution of the hybridization probability. The probability that a segment of length $k$ is hybridized to its complementary partner (Eq. (22) in Appendix A) is strongly peaked at $k=k_{0} \sim \log t$ (see Eq. (23) in Appendix A). Different colors from red to violet correspond to linearly increasing times $t$ since the beginning of the night phase of the cycle.

nights) by the onset of the aggregation regime (Fig. 1c).

\section{B. Major parameters of the model}

In what follows we focus on slow dynamical processes taking place over multiple day/night cycles. The main input parameter from the intra-night kinetics to the multi-cycle dynamics is the hybridization cutoff length $k_{0}$ discussed above. The multi-cycle dynamics can be described in terms of time-averaged ligation and fragmentation rates, $\lambda$ and $\beta$, respectively. We define $\lambda$ as the rate of bond formation provided that the ends of the two substrates are already properly positioned next to each other due to their hybridization with the template. We further assume that the characteristic fragmentation time $1 / \beta$ is much longer than the 
duration of the day-night cycle ensuring the separation between short and long timescales in the problem. Both $\lambda$ and $\beta$ are averaged over the duration of the day-night cycle with the understanding that fragmentation happens continuously throughout the cycle (possibly with different day and night rates), while the ligation only occurs during the night phase. Thus $\lambda$ implicitly depends on relative durations of night and day phases.

Let $C$ be the overall monomer concentration including both free monomers and those bound in all chains. In the case of random sequence composition, the population of heteropolymers is fully characterized by their length distribution $f_{l}$, defined in such a way that $C \cdot f_{l}$ is the concentration of all polymers of length $l$. By this definition $f_{l}$ is subject to the normalization condition $\sum_{l=1}^{\infty} l f_{l}=1$. The fraction of polymers with a specific sequence is then given by $z^{-l} \cdot f_{l}$.

\section{Detailed balance ansatz}

For template-assisted ligation the effective two-polymer merger rate $\mu$ is given by the ligation rate $\lambda$ multiplied by the probability of hybridization of a template $T$ with two substrates $S_{1}$ and $S_{2}$ bring them into end-to-end configuration shown in Fig. 1b. The major step in constructing an approximate analytical solution of the problem is the assumption of a detailed balance between template-assisted ligation and fragmentation in the steady state of the system:

$$
\beta f_{l+m}=\mu f_{l} \cdot f_{m}
$$

Here, the left-hand side describes the rate at which a chain of length $l+m$ breaks into two pieces of lengths $l$ and $m$ correspondingly. Conversely, the right-hand side is the effective merger rate (hybridization + ligation) at which polymers of lengths $l$ and $m$ are joined to form a longer chain of length $l+m$. Note that according to this description the rate at which a polymer breaks into arbitrary two pieces is proportional to its length or rather its number of intra-polymer bonds.

The detailed balance approximation is not a priory justified in driven, non-equilibrium systems such as ours. However, for chains longer than the optimal overlap length $k_{0}$ the probability of hybridization and thus the effective merger rate $\mu$ saturate (see Methods for derivation and details). Once both $\mu$ and $\beta$ are independent of polymers' lengths, our system becomes mathematically equivalent to the well known reversible step-like polymer- 
ization process for which the detailed balance approximation hold true by the virtue of laws of equilibrium thermodynamics. In spite of this superficial similarity, our system remains intrinsically non-equilibrium since the effective merger rate $\mu$ depends on hybridization between templates and substrates cycled through day and night phases as shown in Fig. 1ab. In addition, the Eq. (2) is expected to break down for chains shorter than $k_{0}$.

To validate our mathematical insights, the analytic solution shown below was followed by numerical simulations of the system carried out without the detailed balance approximation. The agreement between our analytical and numerical results for polymers longer than $k_{0}$ confirms the validity of our approach.

The Eq. (2) is satisfied by the exponential length distribution:

$$
f_{l}=(\beta / \mu) \exp (-l / \bar{L}),
$$

where the characteristic chain length, $\bar{L}$, is determined by the normalization condition $\sum_{l=1}^{\infty} l f_{l}=1$ or $(\beta / \mu) \bar{L}^{2}=1$. This result was obtained by replacing the discrete sum with the integral, which works in the limit $\bar{L} \gg 1$ (see Eq. 26 in Appendix B for the exact formula in which this approximation is relaxed). Hence, the characteristic chain length in the steady state exponential distribution is given by

$$
\bar{L}=\sqrt{\frac{\mu}{\beta}}
$$

\section{Onset of autocatalysis}

$\mu$ is an effective two-polymer merger rate proportional to the probability of finding two terminal ends attached to a template followed by ligation. This probability depends on (a) the overall concentration $C$ and the length distribution of potential templates (b) the strength and kinetics of interactions between the complementary segments on a template and its two substrates.

For short overlaps $k \leq k_{0}$ the hybridization probability follows the equilibrium formula: $\sim \exp (k \cdot \epsilon)$. This increase is followed by an abrupt drop for $k>k_{0}$ (see Fig. 2). By neglecting the contribution of overlap lengths longer than $k_{0}$ one gets

$$
\begin{aligned}
\mu= & \lambda\left(\frac{C}{C_{0}}\right)^{2} \sum_{k_{1}=1}^{k_{0}} \exp \left(k_{1} \cdot \epsilon\right) \sum_{k_{2}=1}^{k_{0}} \exp \left(k_{2} \cdot \epsilon\right) . \\
& \cdot \sum_{l=k_{1}+k_{1}}^{\infty}\left(l-k_{1}-k_{2}+1\right) f_{l} .
\end{aligned}
$$


Here $\lambda$ is the bare ligation rate, $k_{1}$ and $k_{2}$ are the overlap lengths between the template and each of the two substrates. We also introduced the reference concentration $C_{0}=\exp \left[-\Delta G_{0} / k_{B} T\right]$ (in molar) absorbing the initiation free energy. The term $\left(C / C_{0}\right)^{2}$ reflects the fact that the template-assisted ligation is a three-body interaction involving two substrates and one template. The last sum in the r.h.s. of the Eq. (5) is equal to the probability of finding a template region of length $k_{1}+k_{2}$ within a longer heteropolymer. It takes into account that a chain of length $l \geq k_{1}+k_{2}$ has $l-k_{1}-k_{2}+1$ sub-sequences of length $k_{1}+k_{2}$. Requirements of sequence complementarity between the template and each of two substrates were absorbed into the definition of $\epsilon$ within the RSA.

Substituting the exponential distribution $f_{l}$ given by the Eq. (3), performing the triple summation in Eq. (5), and neglecting the terms $\sim 1 / \bar{L}$ (but not $\sim k_{0} / \bar{L}$ ) within the exponents approximately gives $\mu=\lambda\left(C / C_{0}\right)^{2} \exp \left(2 k_{0} \cdot(\epsilon-1 / \bar{L})\right) /[1-\exp (-\epsilon)]^{2}$. Substituting this expression into the Eq. (4) results in the self-consistency equation for $\bar{L}$ :

$$
\bar{L} \exp \left(\frac{k_{0}}{\bar{L}}\right)=\frac{C}{C_{0}} \cdot \sqrt{\frac{\lambda}{\beta}} \cdot \frac{\exp \left(k_{0} \epsilon\right)}{1-\exp (-\epsilon)},
$$

(see the Eq. (30) in the Appendix B for a more precise expression derived without the large $\bar{L}$ approximation). The l.h.s. of this equation reaches its minimal value of $e \cdot k_{0}$ at $\bar{L}=k_{0}$. As a result, the equation has solutions only for concentrations $C$ above a certain threshold value given by

$$
C_{\text {down }}=k_{0} C_{0} \sqrt{\frac{\beta}{\lambda}} \exp \left(1-k_{0} \epsilon\right) \cdot(1-\exp (-\epsilon))
$$

For $C$ significantly larger than this threshold, one can neglect the exponential term in the 1.h.s. of the Eq. (6) so that the characteristic polymer length $\bar{L}$ linearly increases with the concentration as

$$
\bar{L}=\frac{C}{C_{0}} \cdot \sqrt{\frac{\lambda}{\beta}} \cdot \frac{\exp \left(k_{0} \epsilon\right)}{1-\exp (-\epsilon)} .
$$

For monomer concentrations $C$ below the threshold we don't expect long heteropolymers to form. This suggests a first-order transition between the regimes dominated by free monomers and that with a self-sustaining population of long heteropolymeric chains.

To verify and refine our predictions we approach this transition from below, starting with the state dominated by monomers i.e. $f_{1} \simeq 1$. We explore the stability of the monomer mixture with respect to formation of dimers. In this limit, the dimer fraction $f_{2}$ obeys the 
following kinetic equation:

$$
\frac{d f_{2}}{d t}=-\beta f_{2}+\lambda\left(\frac{C}{C_{0}}\right)^{2} \exp (2 \epsilon) f_{1}^{2} f_{2}
$$

where the second term in the r.h.s. reflects the fact that a dimer can be formed out of two monomers and this process needs to be catalyzed by a complementary dimer. The critical concentration $C_{u p}$ above which dimers would exponentially self-amplify is given by

$$
C_{u p}=C_{0} \sqrt{\frac{\beta}{\lambda}} \exp (-\epsilon)
$$

Thus we confirm the existence of an instability in a mixture of monomers with respect to template-assisted formation of longer chains. Note that, as expected for a first-order phase transition, the instability threshold $C_{u p}$ (Eq. (10)) approached from below exceeds the instability threshold $C_{\text {down }}$ (Eq. (7)) approached from above. Thus, as expected for a first-order phase transition, the system will be hysteretic for $C_{\text {down }}<C<C_{u p}$.

\section{E. Numerical results}

To check our calculations we carried out the detailed numerical simulations of our sys-

tem. Specifically, we numerically solved a system of coupled kinetic equations describing the template-assisted ligation and fragmentation processes and calculated the steady state distribution $f_{l}$ :

$$
\begin{aligned}
\frac{1}{2 \beta} \dot{f}_{n}= & -\left[\frac{n}{2}+\Gamma^{2} \sum_{m} \mu_{n, m} f_{m}\right] f_{n}+\sum_{m>n} f_{m}+ \\
& +\Gamma^{2} \sum_{m<n} \frac{\left(1+\delta_{n-m, m}\right)}{2} \mu_{m, n-m} f_{m} f_{n-m}
\end{aligned}
$$

Here $\Gamma$ is the dimensionless control parameter of the model proportional to the monomer concentration:

$$
\Gamma=\left(\frac{C}{C_{0}}\right) \sqrt{\frac{\lambda}{\beta}}
$$

and $\mu_{n m}$ is the merger matrix, which itself linearly depends on the distribution $f_{l}$ as described in Eq. (34) and (15) in Appendices A and C. Note, that these simulations (unlike our analytical theory) allow for overlap length dependence of merger rates do not use the detailed balance ansatz. 


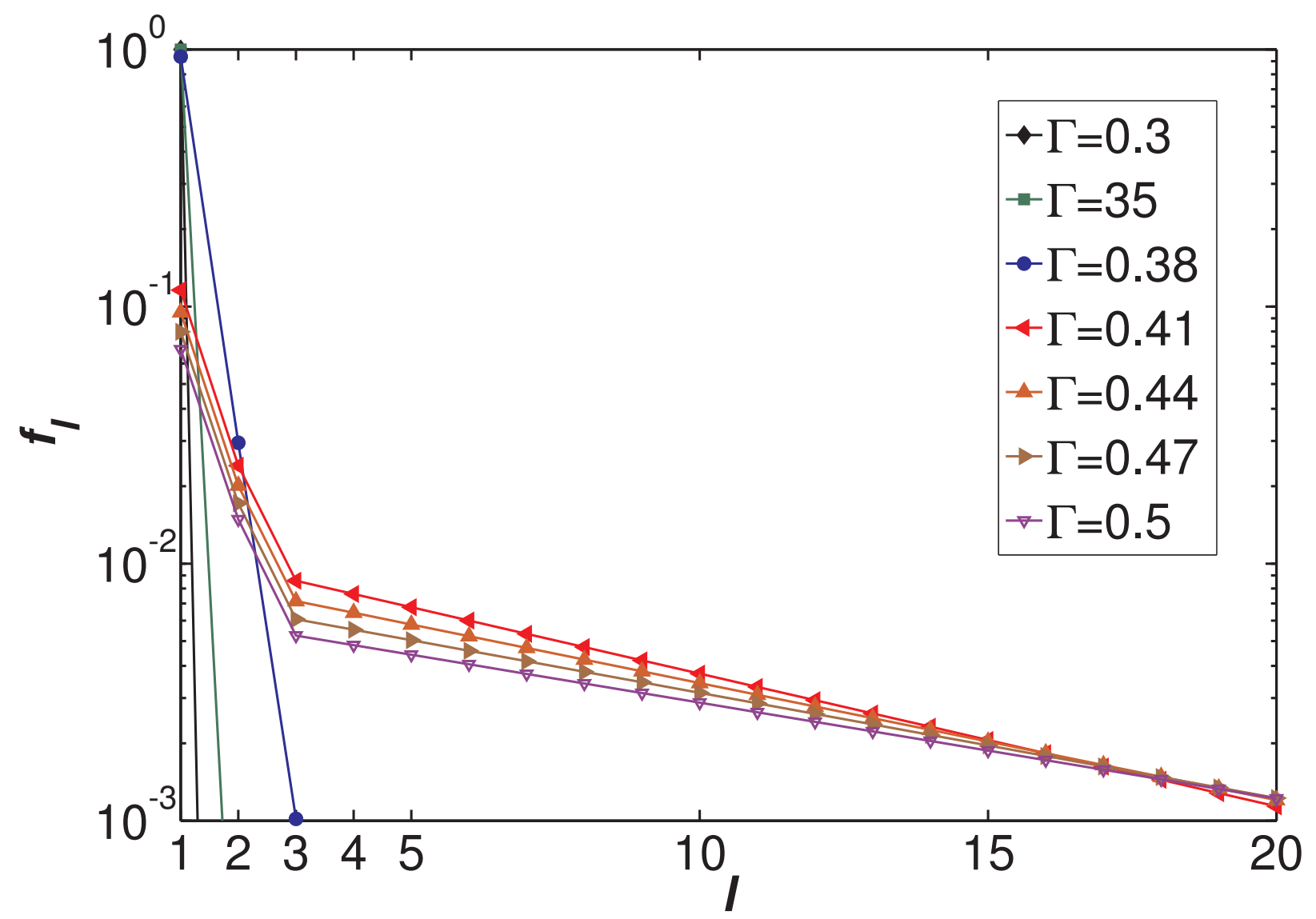

FIG. 3. Chain length distributions. A set of chain length distributions $f_{l}$ plotted for different values of the control parameter $\Gamma=\frac{C}{C_{0}} \sqrt{\frac{\lambda}{\beta}}$ as found by numerical simulations with $k_{0}=3$ and $\epsilon=1$. Distributions in the autocatalytic regime are characterized by long exponentially distributed tails for chains with $l>k_{0}$. Note a sharp transition between monomer-dominated and autocatalytic regimes.

The results of these numerical simulations are in excellent agreement with our analytical calculations. For high enough concentrations $C$ the length distribution $f_{l}$ has a long exponential tail covering the region $l>k_{0}$. Chains of length shorter than $k_{0}$, which do not obey the detailed balance, exhibit a much faster decay as a function of $l$ (see Fig. 3).

Our simulations also confirmed the existence of a first-order transition to a regime dominated by monomers as concentration $C$ was reduced (the red line in Fig. 4). The decay length $\bar{L}$ of the exponential tail of $f_{l}$ for $l \geq k_{0}$ plays the role of the order parameter in this transition. When plotted as a function of concentration $C$ in Fig. 4, it exhibits sharp discontinuities and hysteretic behavior. Our analytical results given by the Eq. (30) (black 


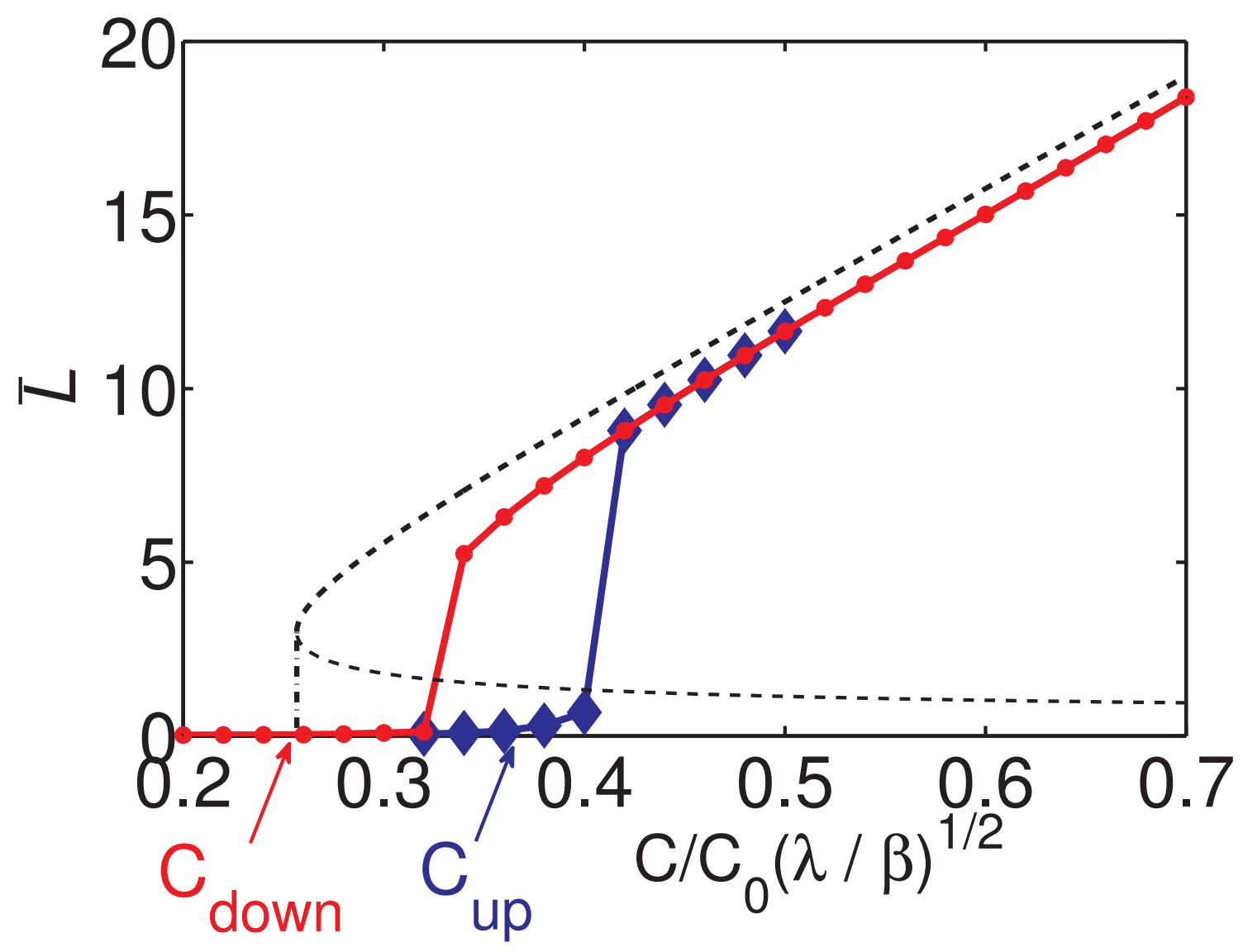

FIG. 4. A hysteretic first order transition between the monomer-dominated and autocatalytic regimes. Different lines/symbols show the characteristic length $\bar{L}$ in our numerical simulations with $k_{0}=3$ for increasing (diamonds), and decreasing (circles) concentration $C$, correspondingly. The dashed line is the prediction of our simplified model given by the Eq. (30). Arrows indicate $C_{u p}$ and $C_{d o w n}$ given by Eqs. (10) and (7) correspondingly.

dashed line in Fig. 4) are in a good agreement with our numerical simulations. The transitions from monomers to long-chained polymers and back in our numerical simulations occur at concentrations somewhat higher than their theoretically predicted values $C_{u p}$ (Eq. 10) and $C_{\text {down }}$ (Eq. 7) marked in Fig. 4 by the blue and red arrows respectively. 


\section{F. Long-night limit}

Our model assumes cyclic changes between "day" and "night" phases. In the beginning of each night phase all polymers are unhybridized, but as time progresses they start forming duplexes of progressively longer lengths. The probability of finding any given segment in a duplex remains low at the early stage of this process. However, if the duration of the night phase is long enough, there would be a time point at which individual polymers would on average have around one hybridized partner. Note that a single polymer may simultaneously have more than one hybridized partner as long as the duplexes with different partners do not overlap with each other. Around this time most polymers in our pool would become immobilized in a gel-like structure schematically depicted in Fig. 1c. At this point the formation of new hybridized complexes effectively stops and the value of $k_{0}$ stops growing. An indirect experimental evidence for such aggregation phase was recently reported by Bellini et $a l .{ }^{16}$.

According to our results, the characteristic chain length $\bar{L}$ given by Eq. (8) exponentially increases with $k_{0}$. In the presence of aggregation this growth is eventually arrested. The upper bound on $\bar{L}$ reached in this case can be determined self-consistently by requiring that individual polymers on average have around one hybridized partner. A chain of length $\bar{L} \gg k_{0}$ contains $\bar{L}-k_{0}+1 \simeq \bar{L}$ segments of length $k_{0}$. The probability of each of these segments to be hybridized at any particular time is $\left(C / C_{0}\right) \cdot \exp \left(k_{0} \cdot \epsilon\right)$. Thus the transition to the aggregated state is expected when

$$
\bar{L} \frac{C}{C_{0}} \exp \left(k_{0} \epsilon\right) \simeq 1
$$

Combining this expression with the Eq. (8) and ignoring the factors of order of 1 one gets the upper bound $\bar{L}_{\max }$ on the characteristic polymer length that could, in principle, be reached by increasing the duration of the night phase:

$$
\bar{L}_{\max } \simeq\left(\frac{\lambda}{\beta}\right)^{\frac{1}{4}} \quad
$$

\section{DISCUSSION}

To summarize, above we considered a general case of random heteropolymers capable of template-assisted ligation. As such our model is applicable to both nucleic acids at the 
dawn of life as well as to artificial self-replicating nano- or micro- structures ${ }^{1,2}$. The major conclusions of our study are as follows. We demonstrated that a population of long chains can be sustained by mutual catalysis sustained exclusively by template-assisted ligation. This state is separated from the monomer-dominated one by a hysteretic first order phase transition (Eqs. $(7,10)$ ) as a function of the concentration. We also demonstrated that the template-assisted ligation in our system is dominated by contributions from templatesubstrate pairs complementary over a well-defined length $k_{0}$, that is kinetically limited. The average length of heteropolymers exponentially increases with $k_{0}$, with the upper bound given by a very simple expression, Eq. (14), depending only on the ratio between the ligation and the breakage rates.

The spontaneous emergence of long polymers demonstrated in our study is of conceptual importance to the long-standing problem of the origin of life. Indeed, we offer a physically plausible path leading from the primordial soup dominated by monomers to a population of sufficiently long self-replicating chains. This transition is one of the least understood processes in the RNA-world hypothesis. It is known that functional RNA-based enzymes (ribozymes) need to be sufficiently long, which makes their spontaneous formation prohibitively unlikely. According to our analysis, both the characteristic chain length and the minimal monomer concentration required for autocatalysis depend on the ratio of ligation and breakage rates. Large values of this ratio $\lambda / \beta \gg 1$ would allow long chains to form at physically possible concentrations $C \ll 1 \mathrm{M}$. One of the reasons that such spontaneous emergence of long-chained polymers has never been observed is that in experimental systems studied so far the ratio $\lambda / \beta$ remained low due to a very slow ligation process ${ }^{17}$. Note that ligation and breakage processes in our system are not direct opposites of each other. Indeed, the ligation of e.g. nucleic acids requires activated terminal bases carrying free energy sufficient to form a new intra-polymer bond. To achieve the conditions necessary for our autocatalytic regime one needs to either use heteropolymers chemically different from modern nucleic acids or to develop new activation pathways different from what has been used in experiments so far. The ligation can be further assisted e.g. by the absorption of polymers onto properly selected crystalline interfaces.

The present study was limited to the simplest version of the problem in which sequences of all heteropolymers were assumed to be completely random. It provides a useful analytically solvable null-model against which future variants can be benchmarked. Even though 
the informational entropy of the pool of polymers in our model is at its maximal value, the template-assisted ligation provides a mechanism for faithful transmission of information to the next generation. We demonstrated that the spontaneous emergence of long chains is possible even in the limit where direct (non-templated) bond formation is negligible. This is especially important since non-templated polymerization is a regular equilibrium phenomenon and as such has a short memory. In contrast, heritable transmission of sequence information via template-assisted ligation opens up an exciting possibility of long-term memory effects and ultimately of the Darwinian evolution in the space of polymer sequences. Incorporation of sequence effects is the logical next step in the development of our model, and we are currently working on it. There are several conceptually distinct yet non mutually exclusive scenarios giving rise to over-representation of certain sequences in the pool of heteropolymers. The first one is driven by the sequence dependence of model parameters such as hybridization free energies, fragmentation and ligation rates, and monomer composition of the primordial soup. The other scenario is the spontaneous symmetry breaking in the sequence space ${ }^{13,18}$. Specifically, our results obtained within the Random Sequence Approximation need to be checked for local and global stability. The local stability analysis deals with small deviations from a state in which populations of all sequences are equal to each other, while the global one perturbs the system by strongly over-representing a small subset of sequences. This can be interpreted, correspondingly, as weak and strong selection limits. Evidence of local or global instability would signal a symmetry breaking and would provide a scenario for the dramatic decrease in informational entropy of the population of polymers. This is analogous to replica symmetry breaking suggested by P.W. Anderson ${ }^{13}$ leading to a population dominated by a relatively small subset of mutually catalyzing sequences.

\section{ACKNOWLEDGEMENTS}

This research used resources of the Center for Functional Nanomaterials, which is a U.S. DOE Office of Science User Facility, at Brookhaven National Laboratory under Contract No. DE-SC0012704. Work at Biosciences Department was supported by US Department of Energy, Office of Biological Research, Grant PM-031. We would like to thank Prof. Mark Lukin, Stony Brook University for valuable discussions. 


\section{APPENDIX A: $k$-MERS AND THEIR HYBRIDIZATION DYNAMICS}

To describe the hybridization dynamics during the night phase we introduce the concept of a $k$-mer defined as the segment of $k$ monomers with the specific sequence $\sigma$ within a longer chain of length $l \geq k$. Let $C \cdot p_{k}^{(\sigma)}$, be the concentration of $k$-mers with particular sequence $\sigma$. Let $C \cdot P_{k}$ be the concentration of all $k$-mers of length $k$, regardless of their sequences. By definition, $P_{k}=\sum_{\sigma} p_{k}^{(\sigma)}$. If all the sequences are completely random, $p_{k}^{(\sigma)}=P_{k} z^{-k}$. Each chain of length $l$ contains $(l+1-k)$ ' $k$-mers', therefore

$$
P_{k}=\sum_{l=k}^{\infty}(l+1-k) f_{l}
$$

Note that $P_{k}$ has the maximum value of 1 which is approached in the limit when all chains are much longer than $k$.

We consider a problem of hybridization of polymers since the start of the night phase of the cycle when all of them are not hybridized. To describe the hybridization kinetics we use the fractions of fully hybridized k-mers $1 \geq \varphi_{k}^{(\sigma)}(t) \geq 0$ as our dynamic variables. By definition, the concentration of such pairs of bound k-mers is $C \cdot p_{k}^{(\sigma)} \varphi_{k}^{(\sigma)}(t)$. We note that hybridization states of different $k$-mers are not independent from each other since some of them overlap. To account for this, we introduce one more variable $\psi_{k}^{(\sigma)} \leq 1-\varphi_{k}^{(\sigma)}$ which is the fraction of all $k$-mers with a given sequence $\sigma$ that are available for hybridization. Now the binding kinetics of all $k$-mers can be described by the following set of coupled kinetic equations:

$$
\tau \dot{\varphi}_{k}^{(\sigma)}=C \cdot p_{k}^{\left(\sigma^{\prime}\right)} \psi_{k}^{\left(\sigma^{\prime}\right)} \psi_{k}^{(\sigma)}-\exp \left(\frac{\Delta G_{\sigma}}{k_{B} T}\right) \varphi_{k}^{(\sigma)}
$$

Here $1 / \tau$ is the hybridization rate, $\Delta G_{\sigma}$ is the hybridization free energy for a given sequence $\sigma$, and $\sigma^{\prime}$ is the sequence complementary to $\sigma$. For simplicity, we consider a symmetric case where mutually complementary $k$-mers have the same fraction, $p_{k}^{(\sigma)}=p_{k}^{\left(\sigma^{\prime}\right)}$. In order to solve these equation, one needs to specify a relationship between fraction of available k-mers $\psi_{k}^{(\sigma)}$ and hybridization probabilities, $\varphi_{k}^{(\sigma)}$, that would take into account mutual overlap of the sequences. However, at early stages the hybridization probability remains sufficiently low, and one can therefore assume $\psi_{k}^{(\sigma)}=\psi_{k}^{\left(\sigma^{\prime}\right)} \approx 1$ in Eq. (16). This results in a set of decoupled equations

$$
\tau \dot{\varphi}_{k}^{(\sigma)}=C \cdot p_{k}^{(\sigma)}-\exp \left(\frac{\Delta G_{\sigma}}{k_{B} T}\right) \varphi_{k}^{(\sigma)}
$$


The solution is the exponential relaxation of hybridization variables $\varphi_{k}^{(\sigma)}$ towards their equilibrium values:

$$
\varphi_{k}^{(\sigma)}(t)=K_{k}^{(\sigma)} p_{k}^{(\sigma)}\left(1-\exp \left(-\frac{t}{\tau_{k}^{(\sigma)}}\right)\right)
$$

In this expression

$$
\begin{gathered}
K_{k}^{(\sigma)}=C \exp \left(-\frac{\Delta G_{\sigma}}{k_{B} T}\right) \\
\tau_{k}^{(\sigma)}=\tau \exp \left(-\frac{\Delta G_{\sigma}}{k_{B} T}\right)
\end{gathered}
$$

The single most important factor that determines the hybridization free energy $\Delta G_{\sigma}$ is the sequence length $k$. For simplicity of the analysis we will replace $K_{k}^{(\sigma)}$ with its sequenceaveraged value:

$$
K_{k}^{(\sigma)} \approx K_{k}=C \exp \left(-\frac{\Delta G_{0}+k \Delta G}{k_{B} T}\right)
$$

This leads to the following result:

$$
\begin{gathered}
\varphi_{k}(t)=C P_{k} z^{-k} \exp \left(-\frac{\Delta G_{0}+k \Delta G}{k_{B} T}\right) . \\
\cdot\left(1-\exp \left[-\frac{t}{\tau} \exp \left(\frac{\Delta G_{0}+k \Delta G}{k_{B} T}\right)\right]\right)
\end{gathered}
$$

As shown in Figure 4 at any given time $t$ this expression is strongly peaked at a single value of $k$, which weakly (logarithmically) depends on time:

$$
\begin{gathered}
k \approx k_{0}(t) \simeq-\frac{k_{B} T}{\Delta G} \log \left(\frac{t}{\tau}\right) \\
\varphi_{k_{0}} \simeq C P_{k_{0}} \exp \left(-\frac{\Delta G_{0}}{k_{B} T}+\varepsilon k_{0}\right)
\end{gathered}
$$

\section{APPENDIX B: EVALUATING THE EFFECTS OF A FINITE $\bar{L}$.}

The equations (3) and (6) in the main text were derived in the limit $\bar{L} \gg 1$. Below we will relax these approximations to derive the exact formula working for arbitrary $\bar{L}$.

In deriving the Eq. (3) in the main text we replaced the discrete summation with an integral. This approximation can be avoided by performing an explicit summation of the discrete geometric progression:

$$
\sum_{l=1}^{\infty} l \cdot \exp \left(-\frac{l}{\bar{L}}\right)=\frac{\exp \left(-\frac{1}{L}\right)}{\left[1-\exp \left(-\frac{1}{L}\right)\right]^{2}}=\frac{1}{4 \sinh \left(\frac{1}{2 L}\right)^{2}}
$$


This amounts to replacing $\bar{L}$ in Eq. (3) with $\frac{1}{2 \sinh \left(\frac{1}{2 L}\right)}$ :

$$
\frac{1}{2 \sinh \left(\frac{1}{2 \bar{L}}\right)}=\sqrt{\frac{\mu}{\beta}}
$$

The exact triple summation of the Eq. (5) in the main text

$$
\begin{array}{r}
\mu=\lambda\left(\frac{C}{C_{0}}\right)^{2} \sum_{k_{1}=1}^{k_{0}} \exp \left(k_{1} \cdot \epsilon\right) \sum_{k_{2}=1}^{k_{0}} \exp \left(k_{2} \cdot \epsilon\right) . \\
\cdot \sum_{l=k_{1}+k_{1}}^{\infty}\left(l-k_{1}-k_{2}+1\right) f_{l}
\end{array}
$$

for $f_{l} \sim \exp (-l / \bar{L})$ can be carried out in two steps. First, the sum over $l$ combined with normalization $\sum_{l} l \cdot f_{l}=1$ gives rise to

$$
\begin{array}{r}
\mu=\lambda\left(\frac{C}{C_{0}}\right)^{2} \exp (1 / \bar{L}) \sum_{k_{1}=1}^{k_{0}} \exp \left[k_{1} \cdot(\epsilon-1 / \bar{L})\right] . \\
\cdot \sum_{k_{2}=1}^{k_{0}} \exp \left[k_{2} \cdot(\epsilon-1 / \bar{L})\right] .
\end{array}
$$

The discrete summation over $k_{1}$ and $k_{2}$ results in

$$
\mu=\lambda\left(\frac{C}{C_{0}}\right)^{2} \exp (1 / \bar{L})\left(\frac{\exp \left[k_{0}(\epsilon-1 / \bar{L})\right]-1}{1-\exp (-\epsilon+1 / \bar{L})}\right)^{2}
$$

The Eq. (6) then becomes

$$
\begin{array}{r}
\frac{1}{2 \sinh \left(\frac{1}{2 \bar{L}}\right)} \exp \left(\frac{k_{0}-1 / 2}{\bar{L}}\right)= \\
=\frac{C}{C_{0}} \cdot \sqrt{\frac{\lambda}{\beta}} \cdot \frac{\exp \left(k_{0} \epsilon\right)-\exp \left(k_{0} / \bar{L}\right)}{1-\exp (-\epsilon+1 / \bar{L})} .
\end{array}
$$

Here we neglected the exponentially small term in the enumerator of the r.h.s. of Eq. (29). The dashed line in Fig. 4 shows $\bar{L}$ defined by this equation plotted as a function of $C$.

\section{APPENDIX C: LIGATION-FRAGMENTATION KINETICS}

The Eq. (6) describes the effective merger rate $\mu$ when lengths $n$ and $m$ of two substrate chains hybridized to a template are longer than $k_{0}$. In a more general case one needs to introduce length-dependent effective merger rate $\mu_{n m}$. Under RSA this rate is given by:

$$
\mu_{n m}=\lambda C^{2} \sum_{k_{1}=1}^{\min \left(n, k_{0}\right)} \sum_{k_{2}=1}^{\min \left(m, k_{0}\right)} \frac{P_{k_{1}+k_{2}}}{z^{k_{1}+k_{2}}}
$$




$$
\begin{aligned}
& \cdot \exp \left(-\frac{2 \Delta G_{0}+\left(k_{1}+k_{2}\right) \cdot \Delta G}{k_{B} T}\right)= \\
& =\lambda\left(\frac{C}{C_{0}}\right)^{2} \cdot \sum_{k_{1}=1}^{\min \left(n, k_{0}\right)} \sum_{k_{2}=1}^{\min \left(m, k_{0}\right)} P_{k_{1}+k_{2}} \cdot \\
& \cdot \exp \left(\left(k_{1}+k_{2}\right) \cdot \epsilon\right)
\end{aligned}
$$

Here $\mu_{n m}$ corresponds to a particular order in which chains $n$ and $m$ merge into a longer chain. Note that for directed chains such as nucleic acids there are two ways of merging chains, while for undirected polymers there are four.

For nucleic acids, when two chain segments are bound to the same template and are directly adjacent to each other (Fig. 1ab) there is an additional gain in free energy $\Delta G_{s t}$ due to stacking. It is straightforward to incorporate $\Delta G_{s t}$ into our formalism by redefining $C_{0}$ as $C_{0}=\exp \left[-\left(\Delta G_{0}+\Delta G_{s t} / 2\right) / k_{B} T\right]$ (in molar).

For directed polymers the resulting set of kinetic equations can be written as:

$$
\begin{aligned}
& \frac{1}{2 \beta} \dot{f}_{n}=-\left[\frac{n}{2}+\Gamma^{2} \sum_{m} \mu_{n, m} f_{m}\right] f_{n}+\sum_{m>n} f_{m}+ \\
& +\Gamma^{2} \sum_{m<n} \frac{\left(1+\delta_{n-m, m}\right)}{2} \mu_{m, n-m} f_{m} f_{n-m}
\end{aligned}
$$

Here $\Gamma$ is the dimensionless control parameter of the model which is proportional to monomer density:

$$
\Gamma=\left(\frac{C}{C_{0}}\right) \sqrt{\frac{\lambda}{\beta}}
$$

and $\mu_{n m}$ is the " $k$-mer"- dependent ligation matrix:

$$
\mu_{n m}=\sum_{k_{1}=1}^{\min \left(n, k_{0}\right)} \sum_{k_{2}=1}^{\min \left(m, k_{0}\right)} P_{k_{1}+k_{2}} \exp \left(\epsilon \cdot\left(k_{1}+k_{2}\right)\right)
$$

This set of kinetic equations gives a complete description of the system in question and was numerically integrated to compare with our analytical results.

\section{REFERENCES}

${ }^{1}$ T. Wang, R. Sha, R. Dreyfus, M. E. Leunissen, C. Maass, D. J. Pine, P. M. Chaikin, and N. C. Seeman, Nature 478, 225 (2011).

2Z. Zeravcic, and M. P. Brenner, Proc. Natl. Acad. Sci. USA 111, 1748 (2014).

${ }^{3}$ M. Eigen and P. Schuster, Naturwissenschaften 64, 541 (1977). 
${ }^{4}$ F. J. Dyson, Journal of Molecular Evolution 18, 344 (1982).

${ }^{5}$ S.A. Kauffman, Journal of Theoretical Biology 119, 1 (1986).

${ }^{6}$ S. Jain and S. Krishna, Phys. Rev. Lett. 81, 5684 (1998).

${ }^{7}$ J. A. Doudna, J. W. Szostak, Nature 339, 519 (1989).

${ }^{8}$ T. A. Lincoln and G. F. Joyce, Science 323, 1229 (2009).

${ }^{9}$ W. Gilbert, Nature 319, 618 (1986).

${ }^{10}$ L. E. Orgel, Critical Reviews in Biochemistry and Molecular Biology 39, 99 (2004).

${ }^{11}$ M. P. Robertson and G. F. Joyce, Cold Spring Harbor Perspectives in Biology 4, a003608 (2012).

${ }^{12}$ C. B. Mast, S. Schink, U. Gerland, and D. Braun, Proc. Natl. Acad. Sci. USA 110, 8030 (2013).

${ }^{13}$ P.W. Anderson, Proc. Natl. Acad. Sci. USA 80, 3386 (1983).

${ }^{14}$ P. W. Anderson, and D. L. Stein, in Self-Organizing Systems edited by F. E. Yates, A. Garfinkel, D. O. Walter, and G. B. Yates, (Springer US, 1987) pp. 445-457.

${ }^{15}$ J. Derr, M. L. Manapat, S. Rajamani, K. Leu, R. Xulvi-Brunet, I. Joseph, M. A. Nowak, and I. A. Chen, Nucleic Acids Research 40, 4711 (2012).

${ }^{16}$ T. Bellini, G. Zanchetta, T. P. Fraccia, R. Cerbino, E. Tsai, G. P. Smith, M. J. Moran, D. M. Walba, and N. A. Clark, Proc. Natl. Acad. Sci. USA 109, 1110 (2012).

${ }^{17}$ R. Rohatgi, D.P. Bartel, J. W. Szostak, J. Am. Chem. Soc. 118, 3332 (1996).

${ }^{18}$ K. Vetsigian and N. Goldenfeld, Proc. Natl. Acad. Sci. USA 106, 215 (2009). 